\title{
Characterization of distributions of $Q$-independent random variables on locally compact Abelian groups
}

\author{
Margaryta Myronyuk
}

Let $X$ be a second countable locally compact Abelian group. We prove some group analogues of the Skitovich-Darmois, Heyde and Kac-Bernstein characterisation theorems for $Q$-independent random variables taking values in the group $X$. The proofs of these theorems are reduced to solving some functional equations on the character group of the group $X$.

Keywords: Characterization theorem, Q-independent random variables, the SkitovichDarmois theorem, the Heyde theorem, the Kac-Bernstein theorem

Mathematics Subject Classification: 60B15 $\cdot 62 \mathrm{E} 10 \cdot 43 \mathrm{~A} 35$

\section{Introduction}

In the article [15] A.M. Kagan and G.J. Székely introduced a notion of $Q$-independence of random variables which generalizes a notion of independence of random variables. They proved that the Cramér theorem about decomposition of Gaussian distribution holds true if instead of independence $Q$-independence is considered. They also proved that the Skitovich-Darmois, Marcinkiewicz and Vershik theorems hold true for $Q$-independent random variables. In the papers [18] and [19] B.L.S. Prakasa Rao considered some generalizations of the Kotlarski theorem and similar results (see [16]) for $Q$-independent random variables.

The series of characterization theorems of mathematical statistics were generalized on different algebraic structures. Especially, much attention has been devoted to the study of analogues of characterization theorems for locally compact Abelian groups (see e.g. ([5]-[11]). Following A.M. Kagan and G.J. Székely [15] in the paper [12] G.M. Feldman in a natural way introduced the notion of $Q$-independence for random variables taking values in a locally compact Abelian group. He proved that if we consider $Q$-independence instead of independence, then the group analogue of the Cramér theorem ([1], [2]), and some group analogues of the the Skitovich-Darmois ([6]) and Heyde theorems ([13]) hold true for the same classes of groups.

In this paper we continue research of characterization theorems for $Q$-independent random variables with values in a locally compact Abelian group $X$ which were started in [12]. We study a group analog of the Skitovich-Darmois theorem for $Q$-independent random variables and linear forms with integer coefficients ([5]). We also study a group analog of the Heyde theorem for $Q$ independent random variables, where coefficients of linear forms are topological automorphisms of the group $X([8])$. We prove that the class of groups $X$ where these theorems hold true does not change if we replace independence for $Q$-independence. In contrast to these results we show that the class of groups $X$ where the Kac-Bernstein theorem ([3]) holds true narrows if we replace the condition of independence for $Q$-independence.

In the paper we suppose that $X$ is a second countable locally compact Abelian group. Denote by $Y$ the character group of $X$, and by $(x, y)$ the value of a character $y \in Y$ at $x \in X$. If $K$ is a closed subgroup of $X$, denote by $A(Y, K)=\{y \in Y:(x, y)=1$ for all $x \in K\}$ its annihilator. Denote by $\operatorname{Aut}(X)$ the group of topological automorphisms of the group $X$, and by $I$ the identity automorphism of a group. If $\alpha \in \operatorname{Aut}(X)$, then the adjoint automorphism $\widetilde{\alpha} \in \operatorname{Aut}(Y)$ is defined as follows $(x, \widetilde{\alpha} y)=(\alpha x, y)$ for all $x \in X, y \in Y$. Note that $\alpha \in \operatorname{Aut}(X)$ if and only if 
$\widetilde{\alpha} \in \operatorname{Aut}(Y)$. Denote by $\mathbb{R}$ the group of real numbers, by $\mathbb{T}=\{z \in \mathbb{C}:|z|=1\}$ the circle group (the one dimensional torus), and by $\mathbb{Z}$ the group of integers. Let $n$ be an integer. Denote by $f_{n}$ the mapping of $X$ into $X$ defined by the formula $f_{n} x=n x$. Put $X_{(n)}=\operatorname{Ker} f_{n}$ and $X^{(n)}=f_{n}(X)$. A group $X$ is called a Corwin group if $X^{(2)}=X$.

If $\xi$ is a random variable with values in the group $X$, then denote by $\mu_{\xi}$ its distribution and by

$$
\widehat{\mu}_{\xi}(y)=\mathbf{E}[(\xi, y)]=\int_{X}(x, y) d \mu_{\xi}(x), \quad y \in Y,
$$

the characteristic function of the distribution $\mu_{\xi}$.

Let $f(y)$ be a function on the group $Y$, and let $h \in Y$. Denote by $\Delta_{h}$ the finite difference operator

$$
\Delta_{h} f(y)=f(y+h)-f(y) .
$$

We remind that a function $f(y)$ on $Y$ is called a polynomial if

$$
\Delta_{h}^{n+1} f(y)=0
$$

for some $n$ and for all $y, h \in Y$. The minimal $n$ for which this equality holds is called the degree of the polynomial $f(y)$.

Let $\xi_{1}, \ldots, \xi_{n}$ be random variables with values in the group $X$. Following [12] we say that the random variables $\xi_{1}, \ldots, \xi_{n}$ are $Q$-independent if the characteristic function of the vector $\left(\xi_{1}, \ldots, \xi_{n}\right)$ can be represented in the form

$$
\begin{aligned}
& \widehat{\mu}_{\left(\xi_{1}, \ldots, \xi_{n}\right)}\left(y_{1}, \ldots, y_{n}\right)=\mathbf{E}\left[\left(\xi_{1}, y_{1}\right) \cdots\left(\xi_{n}, y_{n}\right)\right]= \\
& =\left(\prod_{j=1}^{n} \widehat{\mu}_{\xi_{j}}\left(y_{j}\right)\right) \exp \left\{q\left(y_{1}, \ldots, y_{n}\right)\right\}, \quad y_{j} \in Y,
\end{aligned}
$$

where $q\left(y_{1}, \ldots, y_{n}\right)$ is a continuous polynomial on the group $Y^{n}$. We will also assume that $q(0, \ldots, 0)=0$.

Denote by $\mathrm{M}^{1}(X)$ the convolution semigroup of probability distributions on the group $X$. We remind that a distribution $\gamma \in \mathrm{M}^{1}(X)$ is called Gaussian (see [17, Chapter IV]) if its characteristic function is represented in the form

$$
\widehat{\gamma}(y)=(x, y) \exp \{-\varphi(y)\}, \quad y \in Y,
$$

where $x \in X$, and $\varphi(y)$ is a continuous non-negative function on the group $Y$ satisfying the equation

$$
\varphi(u+v)+\varphi(u-v)=2[\varphi(u)+\varphi(v)], \quad u, v \in Y .
$$

Denote by $\Gamma(X)$ the set of Gaussian distributions on the group $X$. We note that according this definition the generated distributions are Gaussian.

\section{The Skitovich-Darmois theorem for Q-independent random variables}

V. Skitovich and G. Darmois proved one of the most famous characterization theorem of mathematical statistics in which the Gaussian distribution on the real line is characterized by the independence of two linear forms of $n$ independent random variables ([14, Ch. 3]). A group analogue of the Skitovich-Darmois theorem for random variables with values in a locally compact 
Abelian group, where coefficients of linear forms are integer, was considered in [5] (see also [11, $\S 10.7])$. We need the following definition. A set of integers $\left\{a_{j}\right\}$ is said to be admissible for a group $X$ if $X^{\left(a_{j}\right)} \neq\{0\}$ for all $j$. Let $\xi_{j}, j=1,2, \ldots, n, n \geq 2$, be independent random variables with values in $X$. The admissibility of a set $\left\{a_{j}\right\}_{j=1}^{n}$ when considering the linear form $L=a_{1} \xi_{1}+\ldots+a_{n} \xi_{n}$ is a group analogue of the condition $a_{j} \neq 0, j=1,2, \ldots, n$, for the case of $X=\mathbb{R}$. The following result holds.

Theorem A. Let $X$ be a second countable locally compact Abelian group. Let $\left\{a_{j}\right\}_{j=1}^{n}$ and $\left\{b_{j}\right\}_{j=1}^{n}$ be admissible sets of integers for the group $X$. Let $\xi_{1}, \ldots, \xi_{n}$ be independent random variables with values in $X$ and distributions $\mu_{j}$ with non-vanishing characteristic functions. The independence of the linear forms $L_{1}=a_{1} \xi_{1}+\ldots+a_{n} \xi_{n}$ and $L_{2}=b_{1} \xi_{1}+\ldots+b_{n} \xi_{n}$ yields that all $\mu_{j} \in \Gamma(X)$ if and only if either $X$ is a torsion-free group or $X^{(p)}=\{0\}$, where $p$ is a prime number (in the last case all $\mu_{j}$ are degenerate distributions).

We prove that Theorem A remains true if we change the condition of independence of random variables $\xi_{1}, \ldots, \xi_{n}$ and linear forms $L_{1}$ and $L_{2}$ for $Q$-independence. The following statement is valid.

Theorem 1. Let $X$ be a second countable locally compact Abelian group. Let $\left\{a_{j}\right\}_{j=1}^{n}$ and $\left\{b_{j}\right\}_{j=1}^{n}$ be admissible sets of integers for the group $X$. Let $\xi_{1}, \ldots, \xi_{n}$ be $Q$-independent random variables with values in $X$ and distributions $\mu_{j}$ with non-vanishing characteristic functions. The $Q$-independence of the linear forms $L_{1}=a_{1} \xi_{1}+\ldots+a_{n} \xi_{n}$ and $L_{2}=b_{1} \xi_{1}+\ldots+b_{n} \xi_{n}$ yields that all $\mu_{j} \in \Gamma(X)$ if and only if either $X$ is a torsion-free group or $X^{(p)}=\{0\}$, where $p$ is a prime number (in the last case all $\mu_{j}$ are degenerate distributions).

To prove Theorem 1 we need some lemmas. The proof of the following lemma almost literally coincides with the similar lemma of the paper [12].

Lemma 1. Let $X$ be a second countable locally compact Abelian group and $Y$ be its character group. Let $\left\{a_{j}\right\}_{j=1}^{n}$ and $\left\{b_{j}\right\}_{j=1}^{n}$ be sets of integers. Let $\xi_{1}, \ldots, \xi_{n}$ be $Q$-independent random variables with values in $X$ and distributions $\mu_{j}$. The linear forms $L_{1}=a_{1} \xi_{1}+\ldots+a_{n} \xi_{n}$ and $L_{2}=b_{1} \xi_{1}+\ldots+b_{n} \xi_{n}$ are $Q$-independent if and only if the characteristic functions $\widehat{\mu}_{j}(y)$ satisfy the equation

$$
\prod_{i=1}^{n} \widehat{\mu}_{j}\left(a_{j} u+b_{j} v\right)=\left(\prod_{i=1}^{n} \widehat{\mu}_{j}\left(a_{j} u\right) \prod_{i=1}^{n} \widehat{\mu}_{j}\left(b_{j} v\right)\right) \exp \{q(u, v)\}, \quad u, v \in Y,
$$

where $q(u, v)$ is a continuous polynomial on the group $Y^{2}, q(0,0)=0$.

Proof. On the one hand, since the random variables $\xi_{1}, \ldots, \xi_{n}$ are are $Q$-independent, the characteristic function of the vector $\left(L_{1}, L_{2}\right)$ is of the form

$$
\begin{gathered}
\widehat{\mu}_{\left(L_{1}, L_{2}\right)}(u, v)=\mathbf{E}\left[\left(L_{1}, u\right)\left(L_{2}, v\right)\right]=\mathbf{E}\left[\left(a_{1} \xi_{1}+\cdots+a_{n} \xi_{n}, u\right)\left(b_{1} \xi_{1}+\cdots+b_{n} \xi_{n}, v\right)\right]= \\
=\mathbf{E}\left[\left(\xi_{1}, a_{1} u+b_{1} v\right) \cdots\left(\xi_{n}, a_{n} u+b_{n} v\right)\right]= \\
=\left(\prod_{j=1}^{n} \widehat{\mu}_{\xi_{j}}\left(a_{j} u+b_{j} v\right)\right) \exp \left\{q_{1}\left(a_{1} u+b_{1} v, \ldots, a_{n} u+b_{n} v\right)\right\}, \quad u, v \in Y,
\end{gathered}
$$

where $q_{1}\left(y_{1}, \ldots, y_{n}\right)$ is a continuous polynomial on the group $Y^{n}$. 
On the other hand, it follows from the $Q$-independence of $\xi_{1}, \ldots, \xi_{n}$ that

$$
\begin{aligned}
& \widehat{\mu}_{L_{1}}(y)=\mathbf{E}\left[\left(L_{1}, y\right)\right]=\mathbf{E}\left[\left(a_{1} \xi_{1}+\cdots+a_{n} \xi_{n}, y\right)\right]=\left(\prod_{j=1}^{n} \widehat{\mu}_{\xi_{j}}\left(a_{j} y\right)\right) \exp \left\{q_{1}\left(a_{1} y, \ldots, a_{n} y\right)\right\}, \quad y \in Y, \\
& \widehat{\mu}_{L_{2}}(y)=\mathbf{E}\left[\left(L_{2}, y\right)\right]=\mathbf{E}\left[\left(b_{1} \xi_{1}+\cdots+b_{n} \xi_{n}, y\right)\right]=\left(\prod_{j=1}^{n} \widehat{\mu}_{\xi_{j}}\left(b_{j} y\right)\right) \exp \left\{q_{1}\left(b_{1} y, \ldots, b_{n} y\right)\right\}, \quad y \in Y .
\end{aligned}
$$

Assume that the linear forms $L_{1}$ and $L_{2}$ are $Q$-independent. Then the characteristic function of the vector $\left(L_{1}, L_{2}\right)$ can be written in the form

$$
\widehat{\mu}_{\left(L_{1}, L_{2}\right)}(u, v)=\widehat{\mu}_{L_{1}}(u) \widehat{\mu}_{L_{2}}(v) \exp \left\{q_{2}(u, v)\right\}, \quad u, v \in Y,
$$

where $q_{2}(u, v)$ is a continuous polynomial on the group $Y^{2}$. Put

$$
q(u, v)=-q_{1}\left(a_{1} u+b_{1} v, \ldots, a_{n} u+b_{n} v\right)+q_{1}\left(a_{1} u, \ldots, a_{n} u\right)+q_{1}\left(b_{1} v, \ldots, b_{n} v\right)+q_{2}(u, v) .
$$

It follows from the definition of a polynomial on a group that $q(u, v)$ is a continuous polynomial on the group $Y^{2}$. Obviously, (4) follows from (5) -(9). If (44) holds, then (8) follows from (5) -(7), where $q_{2}(u, v)$ is defined by formula (9). Obviously, $q_{2}(u, v)$ is a continuous polynomial on the group $Y^{2}$. Lemma 1 is proved.

Lemma 2 ([1], [2]). Let $X$ be a second countable locally compact Abelian group. Assume that $X$ contains no subgroup topologically isomorphic to the circle group $\mathbb{T}$. Let $\xi_{1}$ and $\xi_{2}$ be independent random variables with values in the group $X$. If the random variable $\xi=\xi_{1}+\xi_{2}$ has a Gaussian distribution, then $\xi_{j}, j=1,2$, are also Gaussian.

Lemma 3 (4]). Let $X$ be a second countable locally compact Abelian group and $Y$ be its character group. Assume that $X$ contains no subgroup topologically isomorphic to the circle group $\mathbb{T}$. Let $f(y)$ be a characteristic function on the group $Y$. If $f(y)$ is of the form

$$
f(y)=\exp \{P(y)\}, \quad y \in Y,
$$

where $P(y)$ is a continuous polynomial, then $P(y)$ is a polynomial of degree $\leq 2$, and $f(y)$ is the characteristic function of a Gaussian distribution on the group $X$.

Proof of Theorem 1. Sufficiency. By Lemma 1, it follows from the conditions of the theorem that the characteristic functions $\widehat{\mu}_{j}(y)$ satisfy equation (44). Put $\nu_{j}=\mu_{j} * \bar{\mu}_{j}$. Then $\widehat{\nu}_{j}(y)=\left|\widehat{\mu}_{j}(y)\right|^{2}>0, y \in Y$. Obviously, the characteristic functions $\widehat{\nu}_{j}(y)$ satisfy the equation

$$
\prod_{i=1}^{n} \widehat{\nu}_{j}\left(a_{j} u+b_{j} v\right)=\left(\prod_{i=1}^{n} \widehat{\nu}_{j}\left(a_{j} u\right) \prod_{i=1}^{n} \widehat{\nu}_{j}\left(b_{j} v\right)\right) \exp \{r(u, v)\}, \quad u, v \in Y,
$$

where $r(u, v)=q(u, v)+\overline{q(u, v)}$ is a real valued continuous polynomial on the group $Y^{2}, r(0,0)=0$.

Assume first that $X$ is a torsion-free group. Then the group $X$ contains no subgroup topologically isomorphic to the circle group $\mathbb{T}$. Therefore, if we prove that $\nu_{j} \in \Gamma(X)$, then applying Lemma 2 we obtain that $\mu_{j} \in \Gamma(X)$. Hence we can assume from the beginning that $\widehat{\mu}_{j}(y)>0$, $y \in Y, j=1,2, \ldots, n, n \geq 2$. 
Set $\varphi_{j}(y)=-\ln \widehat{\mu}_{j}(y)$. We conclude from (4) that

$$
\sum_{j=1}^{n} \varphi_{j}\left(a_{j} u+b_{j} v\right)=P(u)+Q(v)+q(u, v), \quad u, v \in Y,
$$

where

$$
P(u)=\sum_{j=1}^{n} \varphi_{j}\left(a_{j} u\right), \quad Q(v)=\sum_{j=1}^{n} \varphi_{j}\left(b_{j} v\right) .
$$

We use the finite difference method to solve equation (11). Let $h_{n}$ be an arbitrary element of the group $Y$. Substitute $u+b_{n} h_{n}$ for $u$ and $v-a_{n} h_{n}$ for $v$ in equation (11). Subtracting equation (11) from the resulting equation we obtain

$$
\sum_{j=1}^{n-1} \Delta_{l_{n, j}} \varphi_{j}\left(a_{j} u+b_{j} v\right)=\Delta_{b_{n} h_{n}} P(u)+\Delta_{-a_{n} h_{n}} Q(v)+\Delta_{\left(b_{n} h_{n},-a_{n} h_{n}\right)} q(u, v), \quad u, v \in Y,
$$

where $l_{n, j}=\left(a_{j} b_{n}-b_{j} a_{n}\right) h_{n}, j=1,2, \ldots, n-1$. Let $h_{n-1}$ be an arbitrary element of the group $Y$. Substitute $u+b_{n-1} h_{n-1}$ for $u$ and $v-a_{n-1} h_{n-1}$ for $v$ in equation (13). Subtracting equation (13) from the resulting equation we obtain

$$
\begin{gathered}
\sum_{j=1}^{n-2} \Delta_{l_{n-1, j}} \Delta_{l_{n, j}} \varphi_{j}\left(a_{j} u+b_{j} v\right)=\Delta_{b_{n-1} h_{n-1}} \Delta_{b_{n} h_{n}} P(u)+\Delta_{-a_{n-1} h_{n-1}} \Delta_{-a_{n} h_{n}} Q(v)+ \\
+\Delta_{\left(b_{n-1} h_{n-1},-a_{n-1} h_{n-1}\right)} \Delta_{\left(b_{n} h_{n},-a_{n} h_{n}\right)} q(u, v), \quad u, v \in Y,
\end{gathered}
$$

where $l_{n-1, j}=\left(a_{j} b_{n-1}-b_{j} a_{n-1}\right) h_{n-1}, j=1,2, \ldots, n-2$. Arguing as above we get the equation

$$
\begin{gathered}
\Delta_{l_{2,1}} \Delta_{l_{3,1}} \ldots \Delta_{l_{n, 1}} \varphi_{1}\left(a_{1} u+b_{1} v\right)= \\
=\Delta_{b_{2} h_{2}} \Delta_{b_{3} h_{3}} \ldots \Delta_{b_{n} h_{n}} P(u)+\Delta_{-a_{2} h_{2}} \Delta_{-a_{3} h_{3}} \ldots \Delta_{-a_{n} h_{n}} Q(v)+ \\
+\Delta_{\left(b_{2} h_{2},-a_{2} h_{2}\right)} \Delta_{\left(b_{3} h_{3},-a_{3} h_{3}\right)} \ldots \Delta_{\left(b_{n} h_{n},-a_{n} h_{n}\right)} q(u, v), \quad u, v \in Y,
\end{gathered}
$$

where $h_{m}$ is an arbitrary element of the group $Y, l_{m, 1}=\left(a_{1} b_{m}-b_{1} a_{m}\right) h_{m}, m=2,3, \ldots, n$.

Let $h_{1}$ be an arbitrary element of the group $Y$. Substitute $u+b_{1} h_{1}$ for $u$ and $v-a_{1} h_{1}$ for $v$ in equation (15). Subtracting equation (15) from the resulting equation we obtain

$$
\begin{gathered}
\Delta_{b_{1} h_{1}} \Delta_{b_{2} h_{2}} \Delta_{b_{3} h_{3}} \ldots \Delta_{b_{n} h_{n}} P(u)+\Delta_{-a_{1} h_{1}} \Delta_{-a_{2} h_{2}} \Delta_{-a_{3} h_{3}} \ldots \Delta_{-a_{n} h_{n}} Q(v)+ \\
+\Delta_{\left(b_{1} h_{1},-a_{1} h_{1}\right)} \Delta_{\left(b_{2} h_{2},-a_{2} h_{2}\right)} \Delta_{\left(b_{3} h_{3},-a_{3} h_{3}\right)} \ldots \Delta_{\left(b_{n} h_{n},-a_{n} h_{n}\right)} q(u, v)=0, \quad u, v \in Y .
\end{gathered}
$$

Let $h$ be an arbitrary element of the group $Y$. Substitute $u+h$ for $u$ in equation (16) and subtract equation (16) from the resulting equation. We get

$$
\begin{gathered}
\Delta_{h} \Delta_{b_{1} h_{1}} \Delta_{b_{2} h_{2}} \Delta_{b_{3} h_{3}} \ldots \Delta_{b_{n} h_{n}} P(u)+ \\
+\Delta_{(h, 0)} \Delta_{\left(b_{1} h_{1},-a_{1} h_{1}\right)} \Delta_{\left(b_{2} h_{2},-a_{2} h_{2}\right)} \Delta_{\left(b_{3} h_{3},-a_{3} h_{3}\right)} \ldots \Delta_{\left(b_{n} h_{n},-a_{n} h_{n}\right)} q(u, v)=0, \quad u \in Y .
\end{gathered}
$$

Note that if $h$ and $k$ are arbitrary elements of the group $Y$, by the condition

$$
\Delta_{(h, k)}^{l+1} q(u, v)=0, \quad u, v \in Y
$$


for some $l$.

We assumed that $X$ is a torsion-free group, i.e. $X_{(m)}=\{0\}$ for all $m \in \mathbb{Z}, m \neq 0$. This implies that $\overline{Y^{(m)}}=Y$ for all $m \in \mathbb{Z}, m \neq 0$. In particular, $\overline{Y^{\left(a_{j}\right)}}=Y, \overline{Y^{\left(b_{j}\right)}}=Y, j=1,2, \ldots, n$. Hence taking into account that $h$ and $h_{j}$ are arbitrary elements of the group $Y$, it follows from (17) that

$$
\Delta_{h}^{n+1} P(u)+\Delta_{(h, 0)} \Delta_{(h, k)}^{n} q(u, v)=0, \quad u, v \in Y .
$$

We apply to (19) the operator $\Delta_{(h, k)}^{l+1}$. Taking into account (18), we get

$$
\Delta_{h}^{l+n+2} P(u)=0, \quad u \in Y
$$

Thus, $P(y)$ is a continuous polynomial on the group $Y$. Consider the distribution $\gamma=f_{a_{1}}\left(\mu_{1}\right) * \ldots * f_{a_{n}}\left(\mu_{n}\right)$. Then $\widehat{f_{a_{j}}\left(\mu_{j}\right)}(y)=\widehat{\mu}\left(a_{j} y\right), j=1,2, \ldots, n$. We get that

$$
\widehat{\gamma}(y)=\prod_{j=1}^{n} \widehat{\mu}_{j}\left(a_{j} y\right), \quad y \in Y .
$$

Taking into account (12), we have

$$
\widehat{\gamma}(y)=e^{-P(y)}
$$

Since $X$ is a torsion free group, the group $X$ contains no subgroup topologically isomorphic to the circle group $\mathbb{T}$. Hence Lemma 3 implies that $\gamma \in \Gamma(X)$. Then by Lemma 2 we obtain that all $f_{a_{j}}\left(\mu_{j}\right) \in \Gamma(X)$. This implies that every function $\varphi_{j}(y)$ satisfies equation (3) on the set $Y^{\left(a_{j}\right)}$. Since $\overline{Y^{\left(a_{j}\right)}}=Y, j=1,2 \ldots, n$, every function $\varphi_{j}(y)$ satisfies equation (3) on the group $Y$. This means that all $\mu_{j} \in \Gamma(X)$.

Let $X^{(p)}=\{0\}$, where $p$ is a prime number. Then it follows from admissibility of the sets $\left\{a_{j}\right\}_{j=1}^{n}$ and $\left\{b_{j}\right\}_{j=1}^{n}$ for the group $X$ that $f_{a_{j}}, f_{b_{j}} \in \operatorname{Aut}(X), j=1,2, \ldots, n$. We can apply Theorem 1 of [12] and get that all distributions $\mu_{j}$ are Gaussian. Note that the condition $X^{(p)}=\{0\}$ implies that the connected component of zero of the group $X$ is equal to $\{0\}$. Taking into account that the support of a Gaussian distribution is a coset of the connected component of zero of the group $X$, it means that all $\mu_{j}$ are degenerate distributions.

Necessity. Since Q-independent random variables are independent, necessity follows from Theorem A.

\section{The Heyde theorem for Q-independent random variables}

The similar result to the Skitovich-Darmois theorem was proved by C.C. Heyde ([14, §13.4]), where the condition of the independence of two linear forms is replaced by the condition of the symmetry of the conditional distribution of the linear form $L_{2}$ given $L_{1}$. The following group analogue of the Heyde theorem was proved in [8] (see also [11, §16.2]).

Theorem B. Let $X$ be a second countable locally compact Abelian group. Let $\alpha_{j}, \beta_{j}, j=$ $1,2, \ldots, n, n \geq 2$, be topological automorphisms of $X$ such that $\beta_{i} \alpha_{i}^{-1} \pm \beta_{j} \alpha_{j}^{-1} \in \operatorname{Aut}(X)$ for all $i \neq j$. Let $\xi_{1}, \ldots, \xi_{n}$ be independent random variables with values in $X$ and distributions $\mu_{j}$ with non-vanishing characteristic functions. The symmetry of the conditional distribution of the linear form $L_{2}=\beta_{1} \xi_{1}+\ldots+\beta_{n} \xi_{n}$ given $L_{1}=\alpha_{1} \xi_{1}+\ldots+\alpha_{n} \xi_{n}$ implies that all $\mu_{j} \in \Gamma(X)$ if and only if $X$ contains no elements of order 2 . 
We prove that Theorem $\mathrm{B}$ remains true if we change the condition of independence of random variables $\xi_{1}, \ldots, \xi_{n}$ for $Q$-independence. The following statement is valid.

Theorem 2. Let $X$ be a second countable locally compact Abelian group. Let $\alpha_{j}, \beta_{j}, j=$ $1,2, \ldots, n, n \geq 2$, be topological automorphisms of $X$ such that $\beta_{i} \alpha_{i}^{-1} \pm \beta_{j} \alpha_{j}^{-1} \in \operatorname{Aut}(X)$ for all $i \neq j$. Let $\xi_{1}, \ldots, \xi_{n}$ be $Q$-independent random variables with values in $X$ and distributions $\mu_{j}$ with non-vanishing characteristic functions. The symmetry of the conditional distribution of the linear form $L_{2}=\beta_{1} \xi_{1}+\ldots+\beta_{n} \xi_{n}$ given $L_{1}=\alpha_{1} \xi_{1}+\ldots+\alpha_{n} \xi_{n}$ implies that all $\mu_{j} \in \Gamma(X)$ if and only if $X$ contains no elements of order 2 .

To prove Theorem 2 we need the following lemma.

Lemma 4. Let $X$ be a second countable locally compact Abelian group, $Y$ be its character group. Let $\alpha_{j}, \beta_{j}, j=1,2, \ldots, n, n \geq 2$, be topological automorphisms of the group $X$. Let $\xi_{1}, \ldots, \xi_{n}$ be $Q$-independent random variables with values in $X$ and distributions $\mu_{j}$. The conditional distribution of the linear form $L_{2}=\beta_{1} \xi_{1}+\ldots+\beta_{n} \xi_{n}$ given $L_{1}=\alpha_{1} \xi_{1}+\ldots+\alpha_{n} \xi_{n}$ is symmetric if and only if the characteristic functions $\widehat{\mu}_{\xi_{j}}(y)$ satisfy the equation

$$
\prod_{j=1}^{n} \widehat{\mu}_{j}\left(\widetilde{\alpha}_{j} u+\widetilde{\beta}_{j} v\right)=\prod_{j=1}^{n} \widehat{\mu}_{j}\left(\widetilde{\alpha}_{j} u-\widetilde{\beta}_{j} v\right) \exp \{q(u, v)\}, \quad u, v \in Y,
$$

where $q(u, v)$ is a continuous polynomial on the group $Y^{2}, q(0,0)=0$.

Proof. Since the random variables $\xi_{1}, \ldots, \xi_{n}$ are $Q$-independent, the characteristic function of the vector $\left(L_{1}, L_{2}\right)$ is of the form

$$
\begin{gathered}
\widehat{\mu}_{\left(L_{1}, L_{2}\right)}(u, v)=\mathbf{E}\left[\left(L_{1}, u\right)\left(L_{2}, v\right)\right]=\mathbf{E}\left[\left(\alpha_{1} \xi_{1}+\ldots+\alpha_{n} \xi_{n}, u\right)\left(\beta_{1} \xi_{1}+\ldots+\beta_{n} \xi_{n}, v\right)\right] \\
=\mathbf{E}\left[\left(\xi_{1}, \widetilde{\alpha_{1}} u+\widetilde{\beta_{1}} v\right) \ldots\left(\xi_{n}, \widetilde{\alpha_{n}} u+\widetilde{\beta_{n}} v\right)\right] \\
=\prod_{j=1}^{n} \widehat{\mu}_{j}\left(\widetilde{\alpha}_{j} u+\widetilde{\beta}_{j} v\right) \exp \left\{q_{1}\left(\widetilde{\alpha_{1}} u+\widetilde{\beta_{1}} v, \ldots, \widetilde{\alpha_{n}} u+\widetilde{\beta_{n}} v\right)\right\}, \quad u, v \in Y,
\end{gathered}
$$

where $q_{1}\left(y_{1}, \ldots, y_{n}\right)$ is a continuous polynomial on the group $Y^{n}, q_{1}(0, \ldots, 0)=0$. Similarly, the characteristic function of the vector $\left(L_{1},-L_{2}\right)$ is of the form

$$
\widehat{\mu}_{\left(L_{1},-L_{2}\right)}(u, v)=\prod_{j=1}^{n} \widehat{\mu}_{j}\left(\widetilde{\alpha}_{j} u-\widetilde{\beta}_{j} v\right) \exp \left\{q_{1}\left(\widetilde{\alpha_{1}} u-\widetilde{\beta}_{1} v, \ldots, \widetilde{\alpha_{n}} u-\widetilde{\beta_{n}} v\right)\right\}, \quad u, v \in Y
$$

The symmetry of the conditional distribution of the linear form $L_{2}$ given $L_{1}$ means that the random vectors $\left(L_{1}, L_{2}\right)$ and $\left(L_{1},-L_{2}\right)$ are identically distributed, i.e. the characteristic functions (21) and (22) are the same. Put $q(u, v)=-q_{1}\left(\widetilde{\alpha_{1}} u+\widetilde{\beta_{1}} v, \ldots, \widetilde{\alpha_{n}} u+\widetilde{\beta_{n}} v\right)+q_{1}\left(\widetilde{\alpha_{1}} u-\widetilde{\beta_{1}} v, \ldots, \widetilde{\alpha_{n}} u-\widetilde{\beta_{n}} v\right)$. It is obvious that $q(u, v)$ is a continuous polynomial on the group $Y^{2}$, and (20) follows from (21) and (22).

Proof of Theorem 2. Sufficiency. Let $Y$ be the character group of the group $X$. We can put $\zeta_{j}=\alpha_{j} \xi_{j}$ and reduce the proof of the theorem to the case when $L_{1}=\xi_{1}+\ldots+\xi_{n}$ and $L_{2}=\delta_{1} \xi_{1}+\ldots+\delta_{n} \xi_{n}, \delta_{j} \in \operatorname{Aut}(X)$. The conditions $\beta_{i} \alpha_{i}^{-1} \pm \beta_{j} \alpha_{j}^{-1} \in \operatorname{Aut}(X)$ for all $i \neq j$ are transformed into the conditions $\delta_{i} \pm \delta_{j} \in \operatorname{Aut}(X)$ for all $i \neq j$. By Lemma 4 , the symmetry of the 
conditional distribution of the linear form $L_{2}=\delta_{1} \xi_{1}+\ldots+\delta_{n} \xi_{n}$ given $L_{1}=\xi_{1}+\ldots+\xi_{n}$ implies that the characteristic functions $\widehat{\mu}_{j}(y)$ satisfy equation (20) which takes the form

$$
\prod_{j=1}^{n} \widehat{\mu}_{j}\left(u+\widetilde{\delta}_{j} v\right)=\prod_{j=1}^{n} \widehat{\mu}_{j}\left(u-\widetilde{\delta}_{j} v\right) \exp \{q(u, v)\}, \quad u, v \in Y .
$$

Set $\nu_{j}=\mu_{j} * \bar{\mu}_{j}$. Then $\widehat{\nu}_{j}(y)=\left|\widehat{\mu}_{j}(y)\right|^{2}>0, y \in Y$. Obviously, the characteristic functions $\widehat{\nu}_{j}(y)$ satisfy equation

$$
\prod_{j=1}^{n} \widehat{\nu}_{j}\left(u+\widetilde{\delta}_{j} v\right)=\prod_{j=1}^{n} \widehat{\nu}_{j}\left(u-\widetilde{\delta}_{j} v\right) \exp \{r(u, v)\}, \quad u, v \in Y,
$$

where $r(u, v)=q(u, v)+\overline{q(u, v)}$ is a a real valued continuous polynomial on the group $Y^{2}, r(0,0)=$ 0 .

Set $\varphi_{j}(y)=-\ln \widehat{\nu}_{j}(y)$. It follows from (24) that the functions $\varphi_{j}(y)$ satisfy equation

$$
\sum_{j=1}^{n}\left[\varphi_{j}\left(u+\widetilde{\delta}_{j} v\right)-\varphi_{j}\left(u-\widetilde{\delta}_{j} v\right)\right]=r(u, v), \quad u, v \in Y .
$$

We use the finite difference method to solve equation (25).

Let $k_{1}$ be an arbitrary element of the group $Y$. Set $h_{1}=\widetilde{\delta}_{n} k_{1}$, then $h_{1}-\widetilde{\delta}_{n} k_{1}=0$. Substitute $u+h_{1}$ for $u$ and $v+k_{1}$ for $v$ in equation (25). Subtracting equation (25) from the resulting equation we obtain

$$
\sum_{j=1}^{n} \Delta_{l_{1, j}} \varphi_{j}\left(u+\widetilde{\delta}_{j} v\right)-\sum_{j=1}^{n-1} \Delta_{l_{1, j+n}} \varphi_{j}\left(u-\widetilde{\delta}_{j} v\right)=\Delta_{\left(h_{1}, k_{1}\right)} r(u, v), \quad u, v \in Y
$$

where $l_{1, j}=h_{1}+\widetilde{\delta}_{j} k_{1}=\left(\widetilde{\delta}_{n}+\widetilde{\delta}_{j}\right) k_{1}, j=1,2, \ldots, n, l_{1, j+n}=h_{1}-\widetilde{\delta}_{j} k_{1}=\left(\widetilde{\delta}_{n}-\widetilde{\delta}_{j}\right) k_{1}, j=1,2, \ldots, n-$ 1. Let $k_{2}$ be an arbitrary element of the group $Y$. Set $h_{2}=\widetilde{\delta}_{n-1} k_{2}$. Then $h_{2}-\widetilde{\delta}_{n-1} k_{2}=0$. Substitute $u+h_{2}$ for $u$ and $v+k_{2}$ for $v$ in equation (26). Subtracting equation (26) from the resulting equation we obtain

$$
\sum_{j=1}^{n} \Delta_{l_{2, j}} \Delta_{l_{1, j}} \varphi_{j}\left(u+\widetilde{\delta}_{j} v\right)-\sum_{j=1}^{n-2} \Delta_{l_{2, j+n}} \Delta_{l_{1, j+n}} \varphi_{j}\left(u-\widetilde{\delta}_{j} v\right)=\Delta_{\left(h_{2}, k_{2}\right)} \Delta_{\left(h_{1}, k_{1}\right)} r(u, v), \quad u, v \in Y
$$

where $l_{2, j}=h_{2}+\widetilde{\delta}_{j} k_{2}=\left(\widetilde{\delta}_{n}+\widetilde{\delta}_{j}\right) k_{2}, j=1,2, \ldots, n, l_{2, j+n}=h_{2}-\widetilde{\delta}_{j} k_{2}=\left(\widetilde{\delta}_{n}-\widetilde{\delta}_{j}\right) k_{2}, j=$ $1,2, \ldots, n-2$. Arguing as above in $n$ steps we get the equation

$$
\sum_{j=1}^{n} \Delta_{l_{n, j}} \Delta_{l_{n-1, j}} \ldots \Delta_{l_{1, j}} \varphi_{j}\left(u+\widetilde{\delta}_{j} v\right)=\Delta_{\left(h_{n}, k_{n}\right)} \ldots \Delta_{\left(h_{1}, k_{1}\right)} r(u, v), \quad u, v \in Y
$$

where $l_{p, j}=\left(\widetilde{\delta}_{n-p+1}+\widetilde{\delta}_{j}\right) k_{p}, p=1,2, \ldots, n, j=1,2, \ldots, n$. Let $k_{n+1}$ be an arbitrary element of the group $Y$. Set $h_{n+1}=-\widetilde{\delta}_{n} k_{n+1}$, hence $h_{n+1}+\widetilde{\delta}_{n} k_{n+1}=0$. Substitute $u+h_{n+1}$ for $u$ and $v+k_{n+1}$ for $v$ in equation (28). Subtracting equation (28) from the resulting equation we obtain

$$
\sum_{j=1}^{n-1} \Delta_{l_{n+1, j}} \Delta_{l_{n, j}} \Delta_{l_{n-1, j}} \ldots \Delta_{l_{1, j}} \varphi_{j}\left(u+\widetilde{\delta}_{j} v\right)=\Delta_{\left(h_{n+1}, k_{n+1}\right)} \ldots \Delta_{\left(h_{1}, k_{1}\right)} r(u, v), \quad u, v \in Y
$$


where $l_{n+1, j}=h_{n+1}+\widetilde{\delta}_{j} k_{n+1}=\left(\widetilde{\delta}_{j}-\widetilde{\delta}_{n}\right) k_{n+1}, j=1,2, \ldots, n-1$. Equation (29) does not contain the function $\varphi_{n}$. Arguing similarly we sequentially exclude the functions $\varphi_{n-1}, \varphi_{n-2}, \ldots, \varphi_{2}$ from equation (29). Finally we obtain

$$
\Delta_{l_{2 n-1,1}} \Delta_{l_{2 n-2,1}} \ldots \Delta_{l_{1,1}} \varphi_{1}\left(u+\widetilde{\delta}_{1} v\right)=\Delta_{\left(h_{2 n-1}, k_{2 n-1}\right)} \ldots \Delta_{\left(h_{1}, k_{1}\right)} r(u, v), \quad u, v \in Y
$$

where $l_{p, 1}=\left(\widetilde{\delta}_{1}+\widetilde{\delta}_{n-p+1}\right) k_{p}, p=1,2, \ldots, n-1, l_{n, 1}=2 \widetilde{\delta}_{1} k_{n}, l_{n+p, 1}=\left(\widetilde{\delta}_{1}-\widetilde{\delta}_{n-p+1}\right) k_{n+p}, p=$ $1,2, \ldots, n-1$.

Note that if $h$ and $k$ are arbitrary elements of $Y$, then

$$
\Delta_{(h, k)}^{l+1} r(u, v)=0, u, v \in Y
$$

for some $l$. Taking it into account and applying to the both sides of equation (30) the operator $\Delta_{(h, k)}^{l+1}$, we obtain

$$
\Delta_{(h, k)}^{l+1} \Delta_{l_{2 n-1,1}} \Delta_{l_{2 n-2,1}} \ldots \Delta_{l_{1,1}} \varphi_{1}\left(u+\widetilde{\delta}_{1} v\right)=0, \quad u, v \in Y
$$

Taking into account that $k_{p}$ are arbitrary elements of the group $Y$ and $\widetilde{\delta}_{i} \pm \widetilde{\delta}_{j} \in \operatorname{Aut}(Y)$ for $i \neq j$, we can substitute in (31) $l_{n, 1}=2 k, l_{p, 1}=h, p=1,2, \ldots, n-1, n+1, \ldots, 2 n-1$, where $k$ and $h$ are arbitrary elements of the group $Y$. Substituting $v=0$ into the resulting equation we obtain

$$
\Delta_{2 k} \Delta_{h}^{2 n+l-1} \varphi_{1}(u)=0, \quad u \in Y,
$$

where $k, h$ and $u$ are arbitrary elements of $Y$. Since the group $X$ contains no elements of order 2, we have $\overline{Y^{(2)}}=Y$. We deduce from (32) that

$$
\Delta_{h}^{2 n+l} \varphi_{1}(u)=0, \quad u \in Y,
$$

i.e. the function $\varphi_{1}(y)$ is a continuous polynomial. Since the group $X$ contains no elements of order 2, $X$ contains no subgroup topologically isomorphic to the circle group $\mathbb{T}$. By Lemma 3 , $\nu_{1} \in \Gamma(X)$. Applying Lemma 2 we get $\mu_{1} \in \Gamma(X)$. Arguing as above we prove that all $\mu_{j} \in \Gamma(X)$.

Necessity. Since Q-independent random variables are independent, necessity follows from Theorem B.

\section{The Kac-Bernstein theorem for Q-independent identically distributed random variables}

M. Kac and S.N. Bernstein proved that the Gaussian distribution on the real line is characterized by the independence of the sum and the difference of two independent random variables. The following group analogue of the Kac-Bernstein theorem was proved in [3] (see also [11, §9.9]).

Theorem C. Let $X$ be a second countable locally compact Abelian group. Let $\xi_{1}$ and $\xi_{2}$ be independent identically distributed random variables with values in $X$ and distribution $\mu$. The independence of the sum $\xi_{1}+\xi_{2}$ and the difference $\xi_{1}-\xi_{2}$ yields that all $\mu \in \Gamma(X) * I(X)$ if and only if the connected component of zero of the group $X$ contains no more than one element of order 2.

We prove that, unlike Theorems $\mathrm{A}$ and $\mathrm{B}$, Theorem $\mathrm{C}$ does not remain true if we change the condition of independence of random variables $\xi_{1}$ and $\xi_{2}$ and the sum $\xi_{1}+\xi_{2}$ and the difference $\xi_{1}-\xi_{2}$ for $\mathrm{Q}$-independence. In this case the distribution $\mu \in \Gamma(X) * I(X)$ if and only if the 
connected component of zero of the group $X$ contains no elements of order 2 . The following statement is valid.

Theorem 3. Let $X$ be a second countable locally compact Abelian group. Let $\xi_{1}$ and $\xi_{2}$ be $Q$-independent identically distributed random variables with values in $X$ and distribution $\mu$. The $Q$-independence of the sum $\xi_{1}+\xi_{2}$ and the difference $\xi_{1}-\xi_{2}$ yields that $\mu \in \Gamma(X) * I(X)$ if and only if the connected component of zero of the group $X$ contains no elements of order 2.

To prove Theorem 3 we need the following lemmas.

Lemma 5 ([12]). Let $X$ be a second countable locally compact Abelian group. Let $\xi_{1}$ and $\xi_{2}$ be $Q$-independent random variables with values in $X$ and distributions $\mu_{j}$. The $Q$-independence of the sum $\xi_{1}+\xi_{2}$ and the difference $\xi_{1}-\xi_{2}$ yields that all $\mu_{j} \in \Gamma(X) * I(X)$ if and only if the connected component of zero of the group $X$ contains no elements of order 2.

The following lemma was not formulated as a separate statement in the paper [12], but it was essentially proven in it.

Lemma $6([12])$. Let $X=\mathbb{T}$. There exist Q-independent identically distributed random variables $\xi_{1}$ and $\xi_{2}$ with values in $X$ and distribution $\mu$ with a non-vanishing characteristic function such that the sum $\xi_{1}+\xi_{2}$ and the difference $\xi_{1}-\xi_{2}$ are $Q$-independent, whereas $\mu \notin \Gamma(X)$.

Proof of Theorem 3. Sufficiency follows from Lemma 5.

Necessity. Assume that the connected component of zero of the group $X$ contains elements of order 2. Applying [11, Lemma 7.6] for $n=2$ we get that there exists a compact Corwin subgroup $G$ of the group $X$ such that its character group is not a Corwin group. Then by [11, Lemma 7.7] there exists a compact subgroup $K$ of the group $X$ such that the factor-group $X / K$ contains a subgroup $F$ topologically isomorphic to the circle group $\mathbb{T}$. For this reason the distribution $\mu$ on the circle group $\mathbb{T}$ from Lemma 6 can be considered as a distribution on the factor-group $X / K$. We will retain for it the notation $\mu$. Since the character group of the factor-group $X / K$ is topologically isomorphic to the annihilator $A(Y, K)$, we can assume that the characteristic function $\widehat{\mu}(y)$ is defined on $A(Y, K)$. Consider on the group $Y$ the function

$$
h(y)= \begin{cases}\widehat{\mu}(y), & y \in A(Y, K) ; \\ 0, & y \notin A(Y, K) .\end{cases}
$$

Since $A(Y, K)$ is a subgroup and $\widehat{\mu}(y)$ is a positive definite function, the function $h(y)$ is also positive definite function $([11, \S 2.12])$. Since $K$ is a compact group, the annihilator $A(Y, K)$ is an open subgroup. Hence the function $h(y)$ is continuous. By the Bochner theorem there exists a distribution $\lambda \in \mathrm{M}^{1}(X)$ such that $\widehat{\lambda}(y)=h(y)$. Let $\xi_{j}$ be independent identically distributed random variables with values in the group $X$ and distribution $\lambda$.

We verify that the sum $\xi_{1}+\xi_{2}$ and the difference $\xi_{1}-\xi_{2}$ are Q-independent. Indeed, by Lemma 1 , it suffices to show that the characteristic function $\hat{\lambda}(y)$ satisfy equation (4) which takes the form

$$
\widehat{\lambda}(u+v) \widehat{\lambda}(u-v)=\widehat{\lambda}^{2}(u) \widehat{\lambda}(v) \widehat{\lambda}(-v) \exp \{q(u, v)\}, \quad u, v \in Y
$$

where $q(u, v)$ is continuous polynomial on $Y^{2}, q(0,0)=0$.

Let $u, v \in A(Y, K)$. Then it is obvious that (33) holds, because the function $\widehat{\mu}(y)$ satisfy equation (33). If either $u \in A(Y, K), v \notin A(Y, K)$ or $v \in A(Y, K), u \notin A(Y, K)$, then the both sides of equation (33) are equal to zero. If $u, v \notin A(Y, K)$, then the right-hand side of equation (33) is equal to zero. If the left-hand side of equation (33) is not equal to zero, then $u \pm v \in A(Y, K)$. 
This implies that $2 u \in A(Y, K)$. Since $K$ is a compact Corwin group, applying [11, Lemma 7.2] we get that $u \in A(Y, K)$. This contradicts the assumption. Thus the left-hand side of (33) is also equal to zero. We proved that the characteristic function $\hat{\lambda}(y)$ satisfy equation (33). Since $\mu \notin \Gamma(X / K)$, it is obvious that $\lambda \notin \Gamma(X) * I(X)$. Necessity of Theorem 3 is proved.

\section{References}

[1] G.M. Feldman. On the decomposition of Gaussian distributions on groups. Theory Probab. Appl. 22, (1977), 133-140.

[2] G. M. Feldman. Gaussian distributions on locally compact Abelian groups. Teor. Veroyatn. Primen. 23, (1978), 548-563 (Russian), ( English trans. Theory Probab. Appl. 23, (1978), 529-542).

[3] G. M. Feldman. Bernstein Gaussian distributions on groups, (Russian original) Theory Probab. Appl. 31, (1986), 40-49; translation from Teor. Veroyatn. Primen. 31, (1986), 47-58.

[4] G.M. Feldman. Marcinkiewicz and Lukacs theorems on abelian groups, (Russian original) Teor. Veroyatnost. i Primenen. 34 (1989), 330-339; translation in Theory Probab. Appl. 34 (1989), 290-297.

[5] G. M. Feldman. Characterization of the Gaussian distribution on groups by the independence of linear statistics, (Russian original) Siberian Math. J. 31 (1990), 336-345; translation from Sib. Mat. Zh. 31 (1990), 180-190.

[6] G.M. Feldman. A characterization of the Gaussian distribution on abelian groups. Probab. Theory Relat. Fields 126, (2003), 91-102.

[7] G. M. Feldman. On the Heyde theorem for finite Abelian groups. J. of Theoretical Probability 17, (2004), 971-983.

[8] G.M. Feldman. On a characterization theorem for locally compact abelian groups, Probab. Theory Relat. Fields 133 (2005), 345-357.

[9] G. M. Feldman. On the Heyde theorem for discrete Abelian groups. Studia Math. 177, (2006), 67-79.

[10] G. M. Feldman. The Heyde theorem for locally compact Abelian groups. J . of Functional Analysis 258, (2010), 3977-3987.

[11] G.M. Feldman. Functional equations and characterization problems on locally compact abelian groups. EMS Tracts in Mathematics 5, European Mathematical Society (EMS), Zurich, 2008.

[12] G.M. Feldman. Characterization theorems for Q-independent random variables with values in a locally compact abelian group. Aequationes Math. 91, (2017), 949-967.

[13] G.M. Feldman. The Heyde characterization theorem on some locally compact Abelian groups. Theory Probab. Appl. (in Russian) 62, (2017), 499-517. 
[14] A. M. Kagan, Yu. V. Linnik, C.R. Rao. Characterization problems in mathematical statistics (Russian original), Wiley Series in Probability and Mathematical Statistics, John Wiley \& Sons, New York-London-Sydney, 1973 translation from (Kharakterizatsionnye zadachi matematicheskoj statistiki), Nauka, Moskva, 1972.

[15] A.M. Kagan, G. J. Székely. An analytic generalization of independence and identical distributiveness. Statistics and Probability Letters. 110, (2016), 244-248..

[16] I.I. Kotlarski. On characterizing the gamma and the normal distribution, Pacific J. Math., 20, (1967), 69-76.

[17] K.R. Parthasarathy. Probability measures on metric spaces. Probab. Math. Statist. 3. Academic Press, New York - London, 1967.

[18] B. L. S. Prakasa Rao. Characterizations of Probability Distributions Through Linear Forms of Q-Conditional Independent Random Variables, SankhyÏa A: The Indian Journal of Statistics 78, (2016), 221-230.

[19] B. L. S. Prakasa Rao. Characterizations of probability distributions through Q-independence, Teor. Veroyatnost. i Primenen. 62 (2017), 415-420.

B. Verkin Institute for Low Temperature Physics and Engineering of the National Academy of Sciences of Ukraine Kharkiv, Ukraine

myronyuk@ilt.kharkov.ua 